\title{
Analysis on the Fatty Acids and Volatile Components in Pleurotus geesteranus by HS-SPME-GC-MS
}

\author{
Zhenhua Liang, ${ }^{1,2,3}$ Shanei Li, ${ }^{1,4}$ Qiongxin Liang, ${ }^{1}$ Liqiang Ji, ${ }^{1,4}$ Jinmei Wang $\mathbb{D}^{1,2,3}$ \\ and Changqin $\mathrm{Li}^{1}$ \\ ${ }^{1}$ National $R$ \& D Center for Edible Fungus Processing Technology, Henan University, Kaifeng 475004, Henan, China \\ ${ }^{2}$ Functional Food Engineering Technology Research Center, Kaifeng 475004, Henan, China \\ ${ }^{3}$ Joint International Research Laboratory of Food \& Medicine Resource Function, Henan University, Kaifeng 475004, \\ Henan, China \\ ${ }^{4}$ Henan Lonfon Industrial Co., Ltd., Puyang 475300, Henan, China
}

Correspondence should be addressed to Jinmei Wang; wangjinmeiscp@126.com

Received 19 May 2020; Revised 4 June 2020; Accepted 26 August 2020; Published 7 September 2020

Academic Editor: Fatma M. El-Demerdash

Copyright ( 12020 Zhenhua Liang et al. This is an open access article distributed under the Creative Commons Attribution License, which permits unrestricted use, distribution, and reproduction in any medium, provided the original work is properly cited.

\begin{abstract}
The volatile constituents and fatty acids in Pleurotus geesteranus were assayed by headspace solid-phase microextraction coupled with GC-MS. There were 5 volatile compounds in P. geesteranus that accounted for $43.43 \%$ of the total ion current peak area, and its main compounds were 2 -undecanone (13.99\%), 3-ethyl-2,5-dimethyl pyrazine (12.67\%), and l- $\beta$-bisabolene (6.79\%). Fourteen compounds were identified in the ethanol extract of $P$. geesteranus and 6 fatty acids were identified from the petroleum ether extract, which accounted for $93.72 \%$ and $98.48 \%$ of the total ion current peak area, the main compounds in the ethanol extract were ethyl linoleate $(67.36 \%)$ and ethyl palmitate $(21.83 \%)$, and the main fatty acids in the petroleum ether extract were linoleic acid $(78.22 \%)$, palmitic acid $(10.74 \%)$, and oleic acid $(8.13 \%)$.
\end{abstract}

\section{Introduction}

Pleurotus geesteranus, which belongs to the family Pleurotaceae, is native to India $[1,2]$. P. geesteranus contains many types of components including proteins, fat, polysaccharides, vitamins, trace elements, and 8 essential amino acids [3]. The volatile components of edible fungi such as Morchella esculenta [4], P. eryngii [5], Tricholoma matsutake [6], Boletus edulis [7], and Agaricus bisporus [8], which were detected by solid-phase microextraction (SPME), had been reported, and alcohol compounds are the main chemical components of Pleurotus mushrooms. Alcohol and ketone compounds are the key components that affect the flavor of Pleurotus mushrooms. Current studies of $P$. geesteranus mainly focus on cultivation techniques [9], preservation [10], and biological characteristics [11]. A number of research studies report that $P$. geesteranus has antioxidant [12], liver protection [13], antitumor [14], hypolipidemia [15], and antibacterial [16] activities.
However, the valuable medicinal components present in $P$. geesteranus have not been identified yet. In this study, headspace solid-phase microextraction coupled with the gas chromatography-mass spectrometry (HS-SPME-GC/ MS) technique was used to assay the volatile components of P. geesteranus.

\section{Materials and Methods}

2.1. Materials. Dried powder of $P$. geesteranus was provided by Henan Longfeng Industrial Co., Ltd. (Qingfeng, Henan, China) in October 2019.

The GC/MS instrument was an Agilent 7890 gas chromatograph (Agilent Technologies, Santa Clara, CA, USA) equipped with a 5975 mass spectrometer (Agilent Technologies). A solid-phase microextraction (SPME) device (Supelco, USA) and the extraction head were $65 \mu \mathrm{m}$ polydimethylsiloxane (PDMS-DVB, Supelco, USA). C6-C26 n-alkanes was purchased from Alfa Aesar, Haverhill, USA. 
TABLE 1: Compounds and relative percentage of $P$. geesteranus powder.

\begin{tabular}{|c|c|c|c|c|c|}
\hline No. & $\mathrm{RT}(\min )$ & Compound name & Similarity & Relative content (\%) & KI \\
\hline 1 & 9.955 & 3-Ethyl-2,5-dimethyl pyrazine & 90 & 12.67 & 1089 \\
\hline 2 & 14.155 & 2-Undecanone & 94 & 13.99 & 1266 \\
\hline 3 & 16.329 & 2-Phenylcrotonaldehyde & 96 & 6.08 & 1343 \\
\hline 4 & 19.787 & 1- $\beta$-Bisabolene & 95 & 6.79 & 1459 \\
\hline 5 & 21.961 & Cocal & 98 & 3.91 & 1533 \\
\hline Total & & & & 43.43 & \\
\hline
\end{tabular}

2.2. Extraction. $930 \mathrm{~g}$ of $P$. geesteranus powder was taken, and petroleum ether was added to extract 3 times at room temperature for $72 \mathrm{~h}$. After filtration, the filtrate was concentrated by evaporation to obtain the petroleum ether extract with a yield of $1.39 \%$. The residue was extracted with $70 \%$ ethanol at room temperature and then filtered. The filtrate was concentrated by evaporation to obtain the ethanol extract with a yield of $5.37 \%$.

2.3. Methyl Esterification. Petroleum ether extract $(0.5 \mathrm{~g})$ was added into a $10 \mathrm{~mL}$ test tube with petroleum ether/ether $(4 / 3)$ to $5 \mathrm{~mL}$, and then $4 \mathrm{~mL}$ of $0.5 \mathrm{~mol} / \mathrm{L} \mathrm{KOH}-\mathrm{CH}_{3} \mathrm{OH}$ solution was added under $70^{\circ} \mathrm{C}$ water bath for $10 \mathrm{~min}$. After cooling, distilled water $(10 \mathrm{~mL})$ was added to sonicate and centrifuge solutions, and the supernatant was concentrated.

2.4. HS-SPME. A manual SPME device with a fiber precoated with a $65 \mu \mathrm{m}$ thick layer of polydimethylsiloxane/divinylbenzene (PDMS-DVB) was used for extraction. The dry powder of $P$. geesteranus, ethanol extract, and methylated products was placed in $5 \mathrm{~mL}$ vials, and then, the SPME fiber was exposed in the upper space of the sealed vial at $60^{\circ} \mathrm{C}$ for $30 \mathrm{~min}$. After that, the fiber was withdrawn and directly inserted into the GC-MS inlet (temperature $250^{\circ} \mathrm{C}$ ) for $1 \mathrm{~min}$.

2.5. Determination of Fatty Acids and Volatile Components in Pleurotus geesteranus. The fatty acids and volatile constituents were analyzed using the GC/MS instrument. The GC was fitted with a DB-5MS capillary column (30 $\mathrm{m} \times 0.25 \mathrm{~mm} \times 0.25 \mu \mathrm{m}$, Agilent Technologies). Highpurity helium (99.999\%) was used as the carrier gas at a flow rate of $1.0 \mathrm{~mL} / \mathrm{min}$. The inlet temperature was $250^{\circ} \mathrm{C}$. The temperature program was as follows: the initial column temperature was $50^{\circ} \mathrm{C}$ for $2.0 \mathrm{~min}$, then programmed to $120^{\circ} \mathrm{C}$ at a rate of $8^{\circ} \mathrm{C} / \mathrm{min}$, and held for $2 \mathrm{~min}$, and programmed finally to $220^{\circ} \mathrm{C}$ at a rate of $4^{\circ} \mathrm{C} / \mathrm{min}$ and held at $220^{\circ} \mathrm{C}$ for $5 \mathrm{~min}$. Split injection with a split ratio of $10: 1$ was used. The MS was operated in the SCAN mode (m/z 30-400) with electron impact ionization at an ionization energy of $80 \mathrm{eV}$, the ion source temperature was $230^{\circ} \mathrm{C}$, the quadrupole temperature was $150^{\circ} \mathrm{C}$, transmission line temperature was $280^{\circ} \mathrm{C}$, and electron multiplier voltage was $1635 \mathrm{~V}$. According to the previous reports in the literature $[17,18]$, the Kovats retention index (KI) was calculated by using the retention times of $\mathrm{C}_{6}-\mathrm{C}_{26} n$-alkanes that were injected under the same chromatographic conditions. The Kovats retention index calculation formula was as follows:

$$
\mathrm{KI}=100 n+100 \times \frac{t_{R}-t_{R n}}{t_{R n+1}-t_{R n}},
$$

where $n$ and $n+1$ are the number of normal alkane carbon atoms before and after the outflow; $t_{R n}$ and $t_{R n+1}$ are the retention times of the corresponding normal alkane, respectively; and $t_{R}$ is the retention time of the unknown substance in the gas chromatography $\left(t_{R n}<t_{R}<t_{R n+1}\right)$.

\section{Results and Discussion}

According to the above conditions, the components of $P$. geesteranus powder, ethanol extract, and petroleum ether extract were analyzed by GC-MS, and their total ion flow chromatograms were obtained, respectively. The fatty acids and volatile constituents were identified by their mass spectra with the Rtlpest3.L, Nist08.L spectral library, combined with retention index published in the literature [19] and related websites (http://www.vcf-online.nl). Relative percentage amounts of the separated compounds were calculated automatically from peak areas of the total ion chromatograms.

Five compounds accounting for $43.43 \%$ were identified from the powder of $P$. geesteranus, 14 compounds accounting for $93.72 \%$ were identified from ethanol extracts, and 6 fatty acids accounting for $98.48 \%$ were identified from petroleum ether extracts after methylation. The specific results are presented in Table 1 and Table 2.

As shown in Table 1 and Figure 1, the main compounds in $P$. geesteranus powder were 2-undecanone (13.99\%), 3-ethyl-2, 5-dimethylpyrazine (12.67\%), 1- $\beta$-bisabolene (6.79\%), and 2Phenylcrotonaldehyde (6.08\%). Among them, the content of 2undecanone (13.99\%) was the highest. It has been reported that it is not only an important aroma component of P. geesteranus, but also has an insect repellent effect [20]. In addition, 3-ethyl2,5-dimethylpyrazine is a common aroma active substance, and $1-\beta$-bisabolene is mainly used as an edible flavor.

In Table 2 and Figure 2, we could see that the main components in the ethanol extract were ethyl linoleate (67.36\%) and ethyl palmitate $(21.83 \%)$. Compounds in the petroleum ether extract after methylation were linoleate (78.22\%), palmitate (10.74\%), and elaidate (8.13\%) as shown in Table 2 and Figure 3. There were four common compounds: estragole, cis-anethol, elaidate, and linoleate, and the content in the petroleum ether extract was higher than that in the ethanol extract. Among the ethanol extract components, ethyl linoleate had the highest content, and it has a variety of pharmacological effects, such as anti-inflammatory [21], antioxidant [22], and lowering human cholesterol [23]. The relative content of ethyl palmitate in ethanol extracts was also 
TABLE 2: Compounds and relative percentages of ethanol extract and petroleum ether extract.

\begin{tabular}{|c|c|c|c|c|c|c|}
\hline \multirow{2}{*}{ No. } & \multirow{2}{*}{$\mathrm{RT}(\min )$} & \multirow{2}{*}{ Compounds name } & \multicolumn{2}{|c|}{ Relative content (\%) } & \multirow{2}{*}{ Similarity } & \multirow{2}{*}{$\mathrm{KI}$} \\
\hline & & & Ethanol extracts & Methylated products & & \\
\hline 1 & 8.621 & $\gamma$-Terpinene & 0.06 & - & 86 & 1023 \\
\hline 2 & 11.635 & Terpinen-4-ol & 0.30 & - & 96 & 1168 \\
\hline 3 & 11.981 & Benzoic acid & 0.23 & - & 93 & 1184 \\
\hline 4 & 12.821 & Estragole & 0.07 & 0.30 & 98 & 1218 \\
\hline 5 & 15.736 & cis-Anethol & 0.11 & 0.60 & 98 & 1322 \\
\hline 6 & 17.169 & Benzenepropanoic acid & 0.06 & - & 92 & 1371 \\
\hline 7 & 20.677 & trans-Cinnamic acid & 0.18 & - & 97 & 1489 \\
\hline 8 & 22.307 & Niacinamide & 0.16 & - & 95 & 1545 \\
\hline 9 & 28.582 & Pentadecanoate & - & 0.51 & 98 & 1770 \\
\hline 10 & 30.213 & Ethyl pentadecanoate & 1.21 & - & 99 & 1832 \\
\hline 11 & 31.151 & Palmitate & - & 10.74 & 99 & 1868 \\
\hline 10 & 32.782 & Ethyl palmitate & 21.83 & - & 98 & 1933 \\
\hline 11 & 34.956 & Ethyl heptadecanoate & 0.72 & - & 98 & 2023 \\
\hline 12 & 35.598 & Elaidate & 0.14 & 8.13 & 99 & 2050 \\
\hline 13 & 35.845 & Linoleate & 1.30 & 78.22 & 99 & 2061 \\
\hline 14 & 37.476 & Ethyl linoleate & 67.36 & - & 99 & 2130 \\
\hline Total & & & 93.72 & 98.48 & & \\
\hline
\end{tabular}

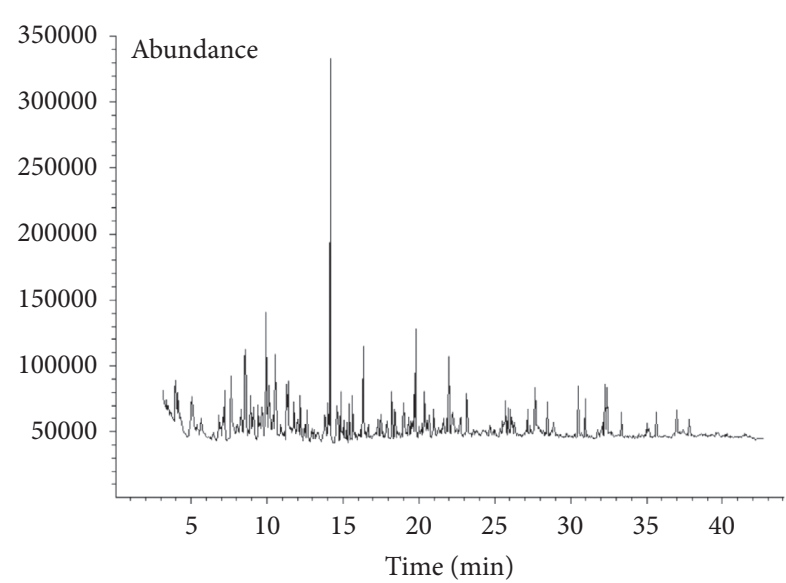

FIGURE 1: Total ion flow chromatogram of volatile components in P. geesteranus powder.

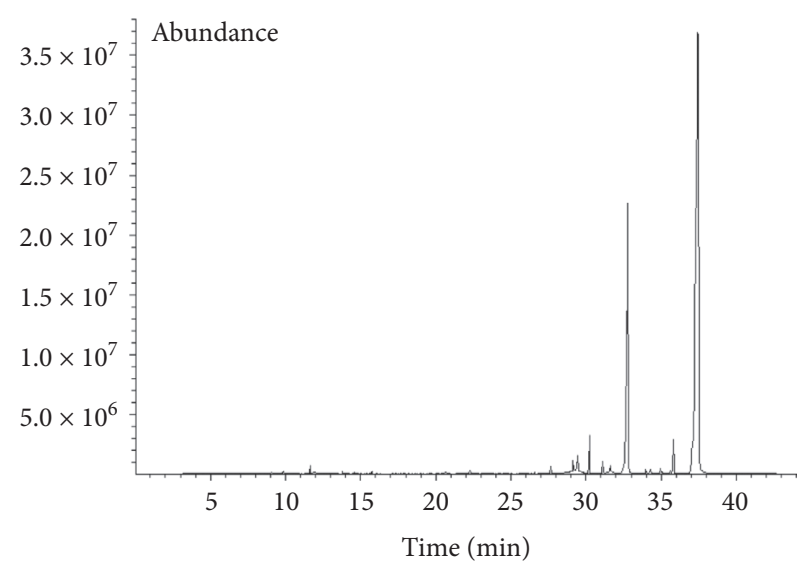

Figure 2: Total ion flow chromatogram of components in ethanol extract.

high, not only as a vasodilator factor for lowering blood pressure [24], but also for preventing nonalcoholic steatohepatitis [25], further having anti-inflammatory [26] and

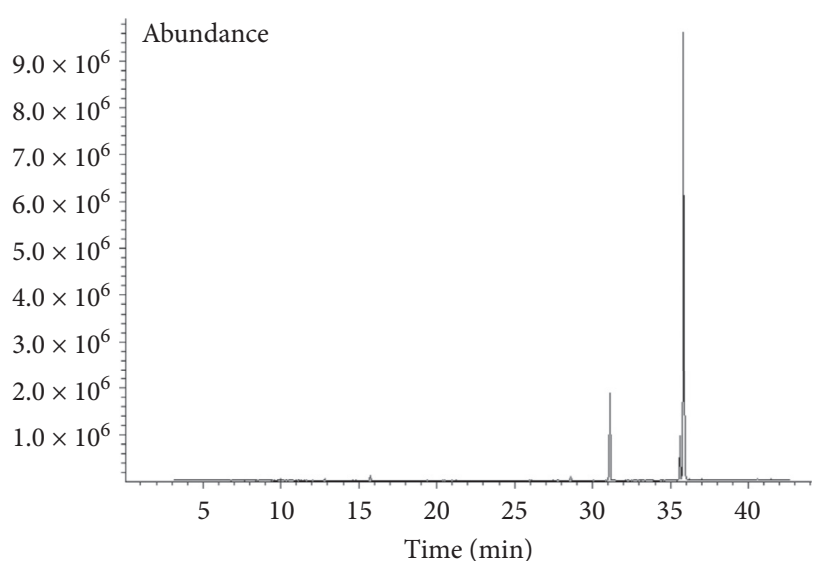

Figure 3: Total ion flow chromatogram of components in petroleum ether extract.

antifibrosis effects [27]. Linoleate was the component with the highest content in petroleum ether extracts and has antiinflammatory [28], antithrombotic [29], anticancer, and antiatherosclerotic effects [30].

Previous studies have found that the main fatty acid components of edible fungi such as Lentinus edodes, Dictyophora indusiata, and Auricularia auricula are linoleic acid, palmitic acid, and linolenic acid $[31,32]$. In this study, we also found that linoleic acid is the main fatty acid in $P$. geesteranus. Liu [33] analyzed the volatile components of $P$. geesteranus by the HP-SPME-GC-MS method, 19 compounds were identified, and the major ones are 3-octanol (55.12\%), 1-octen-3-ol (20.03\%), and 3-octanone (19.22\%), which is different from our research.

\section{Conclusions}

The volatile constituents and fatty acids in $P$. geesteranus were assayed by the HS-SPME-GC-MS method. There were 5 volatile constituents in $P$. geesteranus, 14 compounds were 
identified from the ethanol extract, and 6 fatty acids were identified from the petroleum ether extract. It was found that the main volatile component is 2 -undecanone and the main fatty acid is linoleic acid.

\section{Data Availability}

The data used to support the findings of this study are included within the article.

\section{Conflicts of Interest}

All authors declare that there are no conflicts of interest regarding this study.

\section{Acknowledgments}

This work was funded by the National Key R\&D Program of China (2018YFD0400200) and Key Project in Science and Technology Agency of Henan Province (202102110283).

\section{References}

[1] F. L. Dai, China Fungi Confluence, Science Press, Beijing, China, 1979.

[2] S.-T. Chang, "The world mushroom industry: Trends and technological development," International Journal of Medicinal Mushrooms, vol. 8, no. 4, pp. 297-314, 2006.

[3] H. X. Wang and Q. X. Lan, "Research status of Pleurotus Geesteranus in China," Vegetables, vol. 9, pp. 39-41, 2014.

[4] F. S. Zhang, L. Long, X. R. Yu et al., "Detection and analysis of volatile components in different varieties of morel," Journal of Sichuan University (Natural Science Edition), vol. 56, no. 05, pp. 963-970, 2019.

[5] C. M. Yin, X. Z. Fan, Z. Fan et al., "Analysis of volatile flavor compounds in different Pleurotus species using HS-SPMEGC-MS," Food Science, vol. 39, no. 16, pp. 240-246, 2019.

[6] Y. Guo, D. Chen, Y. Dong, H. Ju, C. Wu, and S. Lin, "Characteristic volatiles fingerprints and changes of volatile compounds in fresh and dried Tricholoma matsutake singer by HS-GC-IMS and HS-SPME-GC-MS," Journal of Chromatography B, vol. 1099, pp. 46-55, 2018.

[7] H. Aisala, J. Sola, A. Hopia, K. M. Linderborg, and M. Sandell, "Odor-contributing volatile compounds of wild edible Nordic mushrooms analyzed with HS-SPME-GC-MS and HS-SPMEGC-O/FID," Food Chemistry, vol. 283, pp. 566-578, 2019.

[8] F. Pei, W. Yang, N. Ma et al., "Effect of the two drying approaches on the volatile profiles of button mushroom (Agaricus bisporus) by headspace GC-MS and electronic nose," LWT - Food Science and Technology, vol. 72, pp. 343-350, 2016.

[9] B. Q. Liu, D. M. Wang, X. L. Zhuo et al., "Pleurotus geesteranus and its cultivation and management application," Xiandai Horticulture, vol. 15, pp. 70-71, 2018.

[10] Z. Zhang, X. Zhang, G. Xin et al., "Umami taste and its association with energy status in harvested Pleurotus geesteranus stored at different temperatures," Food Chemistry, vol. 279, pp. 179-186, 2019.

[11] B. Q. Li, Z. M. Chen, J. Y. Lin et al., "Study on the biological characteristics of Pleurotus geesteranus S3-45 strain," Edible Fungi of China, vol. 36, no. 02, pp. 13-16, 2017.

[12] X. Song, Q. Shen, M. Liu et al., "Antioxidant and hepatoprotective effects of intracellular mycelium polysaccharides from Pleurotus geesteranus against alcoholic liver diseases," International Journal of Biological Macromolecules, vol. 114, pp. 979-988, 2018.

[13] X. Song, Z. Liu, J. Zhang et al., “Antioxidative and hepatoprotective effects of enzymatic and acidic-hydrolysis of Pleurotus geesteranus mycelium polysaccharides on alcoholic liver diseases," Carbohydrate Polymers, vol. 201, pp. 75-86, 2018.

[14] M. Zhang, L. Zhu, S. W. Cui, Q. Wang, T. Zhou, and H. Shen, "Fractionation, partial characterization and bioactivity of water-soluble polysaccharides and polysaccharide-protein complexes from Pleurotus geesteranus," International Journal of Biological Macromolecules, vol. 48, no. 1, pp. 5-12, 2011.

[15] M. Duobin, M. Yuping, G. Lujing, Z. Aijing, Z. Jianqiang, and $\mathrm{X}$. Chunping, "Fermentation characteristics in stirred-tank reactor of exopolysaccharidewith hypolipidemic activity produced by Pleurotus geesteranus 5\#," Anais Da Academia Brasileira De Ciências, vol. 85, no. 4, pp. 1473-1481, 2013.

[16] J. W. Shen, R. R. Wang, C. P. Xu et al., "Study on preparation and bioactivity of sulfated saccharides of Pleurotus geesteranus," Journal of Henan Agricultural Sciences, vol. 43, no. 07, pp. 102-106, 2014.

[17] Y. B. Zhang and W. Y. Kang, "Volatiles in Potentilla discolor by HS-SPME-GC-MS," Chemistry of Natural Compounds, vol. 50, no. 6, pp. 1128-1129, 2014.

[18] Y. Niu, G. Hardy, M. Agarwal, L. Hua, and Y. Ren, "Characterization of volatiles Tribolium castaneum (H.) in flour using solid phase microextraction-gas chromatography mass spectrometry (SPME-GCMS)," Food Science and Human Wellness, vol. 5, no. 1, pp. 24-29, 2016.

[19] L. Sun, W. He, G. Xin et al., "Volatile components, total phenolic compounds, and antioxidant capacities of worminfected Gomphidius rutilus," Food Science and Human Wellness, vol. 7, no. 2, pp. 148-155, 2018.

[20] D. Bisht and C. S. Chanotiya, "2-undecanone rich leaf essential oil from Zanthoxylum armatum," Natural Product Communications, vol. 6, no. 1, pp. 111-114, 2011.

[21] G. D. Liu, X. Bing, D. Huang et al., "Ethyl linoleate inhibits inflammatory reaction induced by titanium particles and its mechanism," Chinese Journal of Tissue Engineering Research, vol. 20, no. 52, pp. 7836-7843, 2016.

[22] T. Masuda, T. Maekawa, K. Hidaka, H. Bando, Y. Takeda, and H. Yamaguchi, "Chemical studies on antioxidant mechanism of curcumin: Analysis of oxidative coupling products from curcumin and linoleate," Journal of Agricultural and Food Chemistry, vol. 49, no. 5, pp. 2539-2547, 2001.

[23] L. Dan and M. Laposata, "Ethyl palmitate and ethyl oleate are the predominant fatty acid ethyl esters in the blood after ethanol ingestion and their synthesis is differentially influenced by the extracellular concentrations of their corresponding fatty acids," Alcoholism: Clinical and Experimental Research, vol. 21, no. 2, pp. 286-292, 1997.

[24] Y.-C. Lee, H.-H. Chang, C.-L. Chiang et al., "Role of perivascular adipose tissue-derived methyl palmitate in vascular tone regulation and pathogenesis of hypertension," Circulation, vol. 124, no. 10, pp. 1160-1171, 2011.

[25] L. Zhang, H.-X. Li, W.-S. Pan et al., "Administration of methyl palmitate prevents non-alcoholic steatohepatitis (NASH) by induction of PPAR- $\alpha$, Biomedicine \& Pharmacotherapy, vol. 111, pp. 99-108, 2019.

[26] N. M. Saeed, E. El-Demerdash, H. M. Abdel-Rahman, M. M. Algandaby, F. A. Al-Abbasi, and A. B. Abdel-Naim, "Anti-inflammatory activity of methyl palmitate and ethyl 
palmitate in different experimental rat models," Toxicology and Applied Pharmacology, vol. 264, no. 1, pp. 84-93, 2012.

[27] M. H. Sharawy, D. S. El-Agamy, A. A. Shalaby, and E.-S. M. Ammar, "Protective effects of methyl palmitate against silica-induced pulmonary fibrosis in rats," International Immunopharmacology, vol. 16, no. 2, pp. 191-198, 2013.

[28] M. Zhao, The Reseach on the Anti-inflammatory Effect of Linoleic Acid and Methyl Linoleate, Southwest Jiaotong University, Chengdu, China, 2012.

[29] K. Yu, X. Q. Huang, S. L. Peng et al., "Research on plant components inhibiting expression of hIL-1 $\beta$ mRNA," West China Journal of Pharmaceutical Sciences, vol. 18, no. 6, pp. 420-421, 2003.

[30] O. Berdeaux, L. Voinot, E. Angioni, P. Juanéda, and J. L. Sébédio, "A simple method of preparation of methyltrans-10, cis-12- andcis-9, trans-11-octadecadienoates from methyl linoleate," Journal of the American Oil Chemists' Society, vol. 75, no. 12, pp. 1749-1755, 1998.

[31] T. Y. Li, J. Y. Yang, X. Fan et al., "Determination of 37 fatty acids in fresh edible mushrooms by gas chromatography coupled with mass spectrometry," Chinese Journal of Health Laboratory Technology, vol. 27, no. 14, pp. 2002-2008, 2017.

[32] Q. Jia, The Reseach on the Chemical Constituents of the Fungus Cap of Dictyophora indusiata and Analysis of Fatty Acid Composition of 10 Edible Fungi in Xi'an, Shaanxi Normal University, Xi'an, China, 2016.

[33] X. Y. Liu, Optimization of Explosion Puffing Drying Process for Pleurotus Geesteranus at Variable Temperature and Pressure Difference and Analyze of Taste Components and Volatile Components, Shenyang Agricultural University, Shenyang, China, 2018. 